\title{
NUTRITION \\ Calcium supplements and osteoporosis: the heart of the matter
}

C alcium supplements with or without vitamin D modestly increase the risk of cardiovascular events, report Bolland et al. in a metaanalysis in which re-analyzed data from the Women's Health Initiative calcium plus vitamin D supplementation study (WHI CaD) were included.

Adequate calcium intake is an established recommendation for the prevention and treatment of osteoporosis, and calcium supplements are in widespread use, often together with vitamin $\mathrm{D}$, amongst elderly individuals. However, the cardiovascular safety of the use of calcium supplements has become a matter of debate over the past few years.

"We originally raised the question of the cardiovascular effects of calcium supplements when we were designing the Auckland Calcium Study, almost a decade ago. Our hypothesis at that time was that calcium supplements may have beneficial effects on cardiovascular events, since we have shown that they produce small benefits to blood cholesterol levels, and because they also reduce blood pressure," says senior author Ian Reid of the University of Auckland. However, Reid and colleagues were surprised to find that the cardiovascular event rate was slightly raised in participants taking calcium.

The team next performed a metaanalysis of the effects of calcium supplements (without co-administered vitamin D) on cardiovascular events, and reported in 2010 that these supplements were associated with an increased risk of myocardial infarction. Nevertheless, questions still remained concerning the cardiovascular effect of taking calcium together with vitamin D.

"The WHI CaD had previously reported that calcium plus vitamin $\mathrm{D}$ did not increase the risk of cardiovascular events," explains Reid. He goes on to explain that the WHI CaD was unusual because it permitted all participants to take personal calcium or vitamin D supplements during the trial. "Self-administration of the trial intervention might have obscured the effects of this intervention."

To test this hypothesis, the researchers assessed the risk of cardiovascular events associated with the use of calcium and vitamin D supplements in the 16,718 women (46\%) from the WHI CaD who were not taking personal calcium supplements at randomization. Among these women, the trial intervention was associated with an increased risk of cardiovascular events-with hazard ratios ranging from 1.13 to 1.22 for cardiovascular events including myocardial infarction and stroke. By contrast, the calcium and vitamin D intervention was not associated with an increased risk of cardiovascular events among women who were taking personal calcium supplements at randomization.

Next, the team conducted a meta-analysis of the cardiovascular effects of the combined intervention with data from three available placebo-controlled trials, including the re-analysed WHI CaD data. Again, modest increases in myocardial infarction and stroke risk were observed. Lastly, the investigators pooled data from this metaanalysis with those from their earlier metaanalysis of calcium monotherapy to establish the effect of calcium with or without vitamin D. Data for 28,072 participants in eight trials were included, of whom 1,384 had an incident myocardial infarction or stroke. A $24 \%$ increased risk of myocardial infarction and a smaller but significant increased risk of stroke were found.

The researchers conclude that the findings warrant a re-assessment of the use of calcium supplements in osteoporosis management. "Since the anti-fracture efficacy of calcium supplements is small and uncertain, we are trading off a significant cardiovascular risk against a very marginal bone benefit," cautions Reid.

But is the current evidence rigorous enough to justify such a re-assessment?

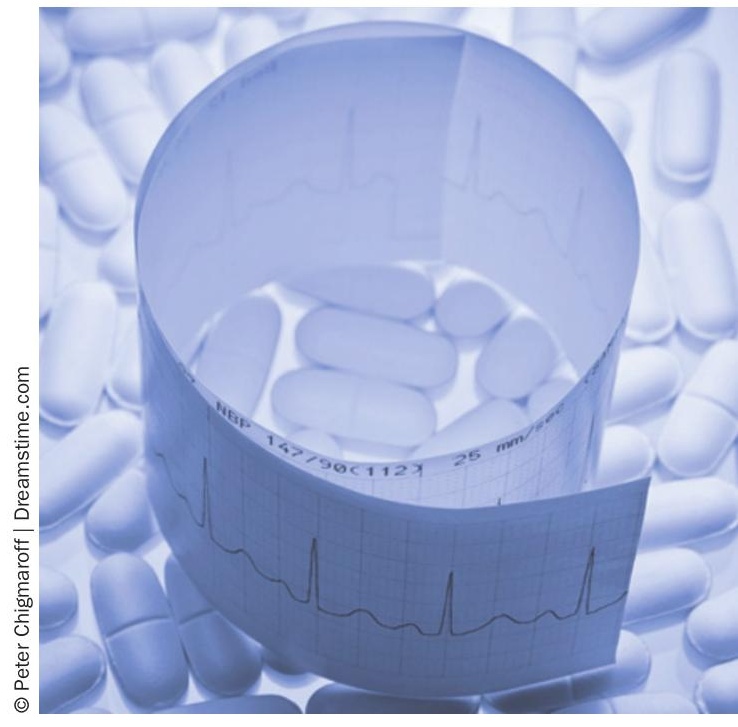

"The methodology is certainly sound but the authors mention the limitations of the study," comments Roger Bouillon of Katholieke Universiteit Leuven, who was not involved in the study. "It is in fact a post hoc analysis and participants who did already take calcium supplementation before the study (nearly 50\%) did not show an increased cardiovascular risk by additional calcium supplements. Furthermore, not mentioned by the authors, compliance was a major problem in the WHI CaD trial and it is unclear to what extend this was analyzed here. In addition, the discussion about calcium supplementation should not be confused nor question the need for adequate supplementation of vitamin D."

"We face a real dilemma because the Institute of Medicine (USA) and most other organizations recommend a daily calcium intake that is higher than that in the usual diet, and calcium and/or vitamin D supplementation are strongly recommended for the prevention and treatment of osteoporosis," points out Bouillon. "If calcium supplementation increases cardiovascular events then the overall benefits are doubtful. A good naturally calcium-rich diet may be a wise solution."

\section{Carol Wilson}

